\title{
Enhanced affective brain representations of chocolate in cravers vs. non-cravers
}

\author{
Edmund T. Rolls and Ciara McCabe \\ University of Oxford, Department of Experimental Psychology, South Parks Road, Oxford OX1 3UD, UK \\ Keywords: human, chocolate, fMRI, affect, craving, orbitofrontal cortex, ventral striatum, pregenual cingulate cortex
}

\begin{abstract}
To examine the neural circuitry involved in food craving, in making food particularly appetitive and thus in driving wanting and eating, we used fMRI to measure the response to the flavour of chocolate, the sight of chocolate and their combination in cravers vs. noncravers. Statistical parametric mapping (SPM) analyses showed that the sight of chocolate produced more activation in chocolate cravers than non-cravers in the medial orbitofrontal cortex and ventral striatum. For cravers vs. non-cravers, a combination of a picture of chocolate with chocolate in the mouth produced a greater effect than the sum of the components (i.e. supralinearity) in the medial orbitofrontal cortex and pregenual cingulate cortex. Furthermore, the pleasantness ratings of the chocolate and chocolaterelated stimuli had higher positive correlations with the fMRI blood oxygenation level-dependent signals in the pregenual cingulate cortex and medial orbitofrontal cortex in the cravers than in the non-cravers. To our knowledge, this is the first study to show that there are differences between cravers and non-cravers in their responses to the sensory components of a craved food in the orbitofrontal cortex, ventral striatum and pregenual cingulate cortex, and that in some of these regions the differences are related to the subjective pleasantness of the craved foods. Understanding individual differences in brain responses to very pleasant foods helps in the understanding of the mechanisms that drive the liking for specific foods and thus intake of those foods.
\end{abstract}

\section{Introduction}

The hedonic effects of food are central to understanding food intake (Rolls, 2005, 2007a). Indeed, the sensory properties of food, including its taste, smell and texture, are rewarding and pleasant when hungry, and become neutral when satiated, and this is related to processing in the orbitofrontal cortex and related areas, as shown by neuronal recordings in macaques (Rolls et al., 1989, 1999; Critchley \& Rolls, 1996) and functional MRI (fMRI) investigations in humans (O’Doherty et al., 2000; Kringelbach et al., 2003; Rolls, 2006, 2007a). These changes in the affective and brain responses to foods appear to be an important part of the mechanism by which food intake is controlled, as shown for example by investigations of sensory-specific satiety (Rolls, 2005, 2007a,b). In this study we investigated differences between chocolate cravers and non-cravers in the hedonic responses to foods, in the amount of food eaten and in the brain responses to foods, in order to examine how differences in brain responses may be related to different appetitive behaviour. As described below, chocolate cravers report that they crave chocolate more than non-cravers, and this is associated with increased liking of chocolate, increased wanting of chocolate and eating chocolate more frequently than non-cravers (Rodriguez et al., 2007).

Previous investigations have shown that the orbitofrontal cortex can be activated by the sight, smell, taste and texture of food, and that the activations in this region are related to the pleasantness of food (Small et al., 2001; Kringelbach et al., 2003; de Araujo \& Rolls, 2004; Wang et al., 2004; Gottfried et al., 2006; Rolls, 2006; McCabe \& Rolls, 2007). We tested the hypothesis that activations to food in the orbitofrontal cortex are different in cravers vs. non-cravers. The

Correspondence: Professor Edmund T. Rolls, as above.

E-mail: Edmund.Rolls@psy.ox.ac.uk

Received 25 January 2007, revised 27 June 2007, accepted 29 June 2007 ventral striatum is implicated in behaviour maintained by conditioned appetitive stimuli, such as a stimulus paired with food or other reinforcers (Everitt, 1997; Cardinal et al., 2002; Kelley, 2003), and we tested the hypothesis that the ventral striatum of humans is activated more by the sight of chocolate in cravers than in non-cravers. The pregenual cingulate cortex is also activated by food stimuli (de Araujo \& Rolls, 2004), and we tested the hypotheses that it is more activated by the sight and/or flavour of food in cravers than non-cravers. The anterior insula contains the primary taste cortex and responds to food in the mouth independently of hunger (Yaxley et al., 1988; Pritchard et al., 1999; Kringelbach et al., 2003; Rolls, 2006), so that it may not represent the positive hedonic value of taste stimuli. We tested the hypothesis that, in contrast to the areas just described, the taste insula may not respond differently to chocolate in cravers and non-cravers. Previous investigations in which brain responses to chocolate were measured did not include a comparison between cravers and noncravers (Small et al., 2001; Kringelbach et al., 2003), and indeed we know of no previous study of brain activations to food in cravers vs. non-cravers of chocolate. An overall aim was to investigate the brain mechanisms that are involved in the sensory and especially affective responses to palatable food and how these may differ between individuals, in order to better understand behavioural choices and the factors that may drive appetite and food intake (Rolls, 2007a).

\section{Materials and methods Overall design}

We compared brain responses to chocolate in a group of cravers vs. a group of non-cravers. To measure the effects of the flavour of chocolate alone, one condition was the delivery of chocolate into the 
mouth (choc). To measure the effects of the sight of chocolate alone, a second condition was the sight of dark chocolate, with no flavour delivered to the mouth (chocpic). To measure the effects of the sight and flavour of chocolate combined, a third condition was the sight of dark chocolate paired with the delivery of chocolate into the mouth (chocd). This condition allowed a supralinearity analysis of whether the activations to the combination of the sight and flavour of chocolate were greater than the sum of the activations produced by the sight alone and by the flavour alone of chocolate. To obtain evidence on how important the sight of a generally preferred dark, i.e. milk, chocolate is vs. the sight of a generally less preferred white chocolate a fourth condition was the presentation of the sight of white chocolate paired with the delivery of chocolate into the mouth (chocw). [Dark, i.e. in this study milk, chocolate may be generally preferred due to the taste and flavour of the cocoa solids that it contains (Michener \& Rozin, 1994).] A fifth condition was the flavour of condensed milk (cmilk), included to test whether any differences in the brain activations of chocolate vs. non-chocolate cravers were especially evident for the craved food, chocolate, or occurred for other foods.

\section{Stimuli}

The main oral stimulus was fine chocolate formulated to be liquid at room temperature; a list of the five stimulus conditions described above is given in Table 1. A control tasteless solution (containing the main ionic components of saliva, $25 \mathrm{~mm} \mathrm{KCl}+2.5 \mathrm{~mm} \mathrm{NaHCO}_{3}$ ) was used as a rinse between trials (tl in Table 1) and, when subtracted from the effects of the other stimuli, allowed somatosensory and any mouth movement effects to be subtracted from the effects produced by the other oral stimuli (O'Doherty et al., 2001; de Araujo et al., 2003a). This is an important control condition that we have pioneered to allow taste, texture and olfactory areas to be shown independently of any somatosensory effects produced by introducing a fluid into the mouth (O'Doherty et al., 2001; de Araujo et al., 2003a,b; de Araujo \& Rolls, 2004). A comparison stimulus was condensed milk. Both the liquid chocolate and condensed milk were diluted with three parts of milk to produce approximately the same sweetness and texture, and to enable them to pass freely through the Teflon delivery tubes.

\section{Experimental design}

The effects in the experiment were measured by psychophysical ratings of pleasantness, intensity and wanting for chocolate made in every trial by the subjects during the fMRI experiments; by fMRI contrasts showing the effects of chocolate in the mouth, chocolate in the mouth accompanied by either the sight of dark chocolate or the

TABLE 1. Stimuli and abbreviations

Chocolate in the mouth only + grey visual stimulus

Picture of dark chocolate only

Chocolate in the mouth + a picture of dark chocolate

Chocolate in the mouth + a picture of white chocolate

Condensed milk in the mouth + grey visual stimulus

Tasteless rinse and control solution + grey visual stimulus sight of white chocolate, the sight of dark chocolate, and the oral delivery of condensed milk; and by fMRI correlation analyses between each of the three psychophysical ratings made throughout the experiment and the brain activations produced by the stimuli being delivered. The details of each stimulus condition are shown in Table 1. If a picture of chocolate was not required as part of the design in a particular trial, a grey image of approximately the same intensity was shown at the corresponding time as indicated in Table 1 to provide a control for the presentation of visual stimuli. The statistical parametric mapping (SPM) design allowed these effects to be compared in the group of chocolate cravers vs. the group of non-cravers.

The experimental protocol consisted of an event-related interleaved design using, in random permuted sequence, the five stimuli described above and shown in Table 1. This number of stimuli was chosen to be feasible given the number of repetitions of each stimulus needed and the length of time that subjects were in the magnet, but at the same time to allow the analyses described above to be made. Stimuli were delivered to the subject's mouth through three Teflon tubes (one for the tasteless rinse control described below) that were held between the lips. Each Teflon tube of approximately $3 \mathrm{~m}$ in length was connected to a separate reservoir via a syringe and a one-way syringe-activated check valve (Model 14044-5, World Precision Instruments, Inc.), which allowed $0.75 \mathrm{~mL}$ of any stimulus to be delivered manually at the time indicated by the computer.

At the beginning of each trial, one of the five stimuli chosen by random permutation was presented. If the trial involved an oral stimulus, this was delivered in a $0.75 \mathrm{~mL}$ aliquot to the subject's mouth. At the same time, at the start of the trial, a visual stimulus was presented, which was either the picture of dark chocolate, the picture of white chocolate or a grey control image of approximately the same intensity. The image was turned off after $7 \mathrm{~s}$, at which time a small green cross appeared on a visual display to indicate to the subject to swallow what was in the mouth. After a delay of $2 \mathrm{~s}$, the subject was asked to rate each of the stimuli for pleasantness in that trial (with +2 being very pleasant and -2 very unpleasant), for intensity in that trial $(0$ to +4$)$ and for current wanting for chocolate $(+2$ for wanting chocolate very much, 0 for neutral and -2 for very much not wanting chocolate). The ratings were made with a visual analogue rating scale in which the subject moved the bar to the appropriate point on the scale using a button box. Each rating period was $5 \mathrm{~s}$ long. After the last rating the grey visual stimulus indicated the delivery of the tasteless control solution that was also used as a rinse between stimuli; this was administered in exactly the same way as a test stimulus and the subject was cued to swallow after $7 \mathrm{~s}$ by the green cross. The tasteless control was always accompanied by the grey visual stimulus. In trials in which only the picture of chocolate was shown, there was no rinse but the grey visual stimulus was shown, in order to allow an appropriate contrast as described below. There was then a 2 s delay period similar to other trials that allowed for swallowing followed by a $1 \mathrm{~s}$ gap until the start of the next trial. A trial was repeated for each of

Chocolate in the mouth, intraoral delivery through a Teflon tube of $0.75 \mathrm{~mL}$ of a specially prepared fine liquid (brown, i.e. milk) chocolate, which was identical for all such trials and could not be seen by the subject; condensed milk in the mouth, intraoral delivery of $0.75 \mathrm{~mL}$ of condensed milk through a tube that also could not be seen by the subject; picture of dark chocolate, a picture of a bar of brown, i.e. milk, chocolate shown on the display screen; picture of white chocolate, a picture of a bar of white chocolate. the five stimulus conditions shown in Table 1 and the whole cycle was repeated nine times. The instruction given to the subject was (in oral delivery trials) to move the tongue once as soon as a stimulus or tasteless solution was delivered (at the time when the grey visual stimulus was turned on) in order to distribute the solution round the mouth to activate the receptors for taste and smell, and then to keep still for the remainder of the $7 \mathrm{~s}$ period until the green cross was shown, when the subject could swallow. This procedure has been shown to allow taste effects to be demonstrated clearly with fMRI, using the procedure of subtracting any activations produced by the tasteless control from those produced by a taste or other stimulus

(choc)
(chocpic)
(chocd)
(chocw)
(cmilk)
(tl)
$.75 \mathrm{~mL}$ of
was iden-
ed milk in
tube that
cture of a

(chocpic) (chocw) (cmilk)

Federation of European Neuroscience Societies and Blackwell Publishing Ltd 
(O’Doherty et al., 2001; de Araujo et al., 2003a,b; de Araujo \& Rolls, 2004). The contrasts reported below had the tasteless control condition subtracted (except for the chocpic condition).

\section{Subjects}

Sixteen healthy volunteers (all females) participated in the study, eight cravers and eight non-cravers. Ethical approval (Central Oxford Research Ethics Committee) and written informed consent from all subjects were obtained before the experiment. The participants were recruited by advertisement specifically requesting either chocolate cravers or chocolate non-cravers. Their status as chocolate cravers or non-cravers was measured with a questionnaire containing questions based on those found in the CCQ-T (chocolate cravers questionnaire) (Cepeda-Benito et al., 2003). The questions were as follows. (1) On a scale from 1 to 10 how much would you say that you sometimes crave chocolate? (2) On a scale from 1 to 10 how much would you say that you like chocolate? (3) On a scale from 1 to 10 how much would you say that you like milk? (4) How frequently do you eat chocolate? (5) How much chocolate do you eat at a time? (To estimate this, please use as units the bar of chocolate shown to you.) (6) Do you crave any other food? If so, what is that food and on a scale from 1 to 10 how much would you say that you crave that food? Questions 1, 2 and 4 contributed 10 points to the chocolate craving measure that we used, the final value of which was divided by 10 to make the score in the range $0-10$. (For question 4,10 points corresponded to the highest value of any of the participants, more than once per day, 9 points to once per day and 1 for less than once per week.) The mean score across all of the questions was $8.14 \pm 1.51$ (SD) for the chocolate cravers and $3.13 \pm 0.94$ for the chocolate non-cravers $(t=7.82$, d.f. $=14, P<0.001)$. There was no overlap in the scores of the cravers and non-cravers, with a score of 5 separating the two groups.

The responses to the questionnaire showed that the chocolate cravers ate chocolate more frequently and ate more chocolate than non-cravers. In particular, the mean score on question 4 of our chocolate questionnaire (How frequently do you eat chocolate?) was $7.4 \pm 1.02$ for cravers and $1.25 \pm 0.44$ for non-cravers (mean \pm SEM, maximum score 10, with the scoring as described in Materials and methods). Moreover, the mean amount of chocolate eaten per week was $370 \pm 154 \mathrm{~g}$ for cravers and $22 \pm 8 \mathrm{~g}$ for non-cravers (mean $\pm \mathrm{SEM}$, Mann-Whitney $\mathrm{U}, P<0.001$ ). The mean score on question 1 of our chocolate questionnaire (On a scale from 1 to 10 how much would you say that you sometimes crave chocolate?) was $7.86 \pm 0.63$ for cravers and $2.13 \pm 0.47$ for non-cravers (mean \pm SEM) $\left(t=7.58\right.$, d.f. $\left.=13, P=4 \times 10^{-6}\right)$. The mean score on question 2 of our chocolate questionnaire (On a scale from 1 to 10 how much would you say that you like chocolate?) was $9.14 \pm 0.40$ for cravers and $6.13 \pm 0.51$ for non-cravers (mean $\pm \mathrm{SEM}$ ) $\left(t=4.73\right.$, d.f. $\left.=13, P=4 \times 10^{-4}\right)$. Thus, most subjects liked chocolate (question 2) and the difference between the groups was much larger for question 1 about the craving for chocolate. [Indeed, the interaction term in comparing craving vs. liking in the two groups, cravers and non-cravers, is highly significant $\left(F_{1,13}=13.7\right.$, $P=0.0002)$, showing that it is especially in their answer to the question about whether they crave chocolate that they are different from the group termed non-cravers.] In this sense, chocolate cravers are a self-identifying group based on their responses to questions about chocolate craving (Rodriguez et al., 2007). [For comparison, the mean score on question 3 of our questionnaire (On a scale from 1 to 10 how much would you say that you like milk?) was $3.11 \pm 1.18$ for cravers and $5.31 \pm 1.14$ for non-cravers (mean \pm SEM).]
The participants were instructed not to eat chocolate for 2 days beforehand and to eat only a small lunch on the day of scanning. Scanning took place at $14.30-16.00 \mathrm{~h}$. The participants were students or post-doctoral scientists aged 20-30 years. The mean body mass index of the cravers was 22.1 and that of the non-cravers was 23.0 (n.s.).

\section{fMRI data acquisition}

Images were acquired with a $3.0 \mathrm{~T}$ VARIAN/SIEMENS whole-body scanner at the Centre for Functional Magnetic Resonance Imaging at Oxford, where T2*-weighted echo planar imaging (EPI) slices were acquired every $2 \mathrm{~s}(\mathrm{TR}=2)$. We used the techniques that we have developed over a number of years (e.g. O'Doherty et al., 2001; de Araujo et al., 2003a) and, as described in detail by Wilson et al. (2002), we carefully selected the imaging parameters in order to minimize susceptibility and distortion artefact in the orbitofrontal cortex. The relevant factors include imaging in the coronal plane, minimizing voxel size in the plane of the imaging, as high a gradient switching frequency as possible $(960 \mathrm{~Hz})$, a short echo time of $25 \mathrm{~ms}$ and local shimming for the inferior frontal area.

Coronal slices (25) with in-plane resolution of $3 \times 3 \mathrm{~mm}$ and between-plane spacing of $4 \mathrm{~mm}$ were obtained with a TR of $2 \mathrm{~s}$. The matrix size was $64 \times 64$ and the field of view was $192 \times 192 \mathrm{~mm}$. Continuous coverage was obtained from $+56(\mathrm{~A} / \mathrm{P})$ to $-50(\mathrm{~A} / \mathrm{P})$. Acquisition was carried out during the task performance yielding 810 volumes in total. A whole brain T2*-weighted EPI volume of the above dimensions, and an anatomical $\mathrm{T} 1$ volume with coronal plane slice thickness of $3 \mathrm{~mm}$ and in-plane resolution of $1.0 \times 1.0 \mathrm{~mm}$ was also acquired.

\section{fMRI data analysis}

The imaging data were analysed using SPM2 (Wellcome Institute of Cognitive Neurology). Pre-processing of the data used SPM2 realignment, reslicing with sinc interpolation, normalization to the Montreal Neurological Institute coordinate system (Collins et al., 1994) used throughout this study, and spatial smoothing with a $8 \mathrm{~mm}$ full width at half maximum isotropic Gaussian kernel and global scaling. The time series at each voxel were low-pass filtered with a haemodynamic response kernel. The time series non-sphericity at each voxel was estimated and corrected for (Friston et al., 2002) and a highpass filter with a cut-off period of $128 \mathrm{~s}$ was applied. In the single event design, a general linear model was then applied to the time course of activation where stimulus onsets were modelled as single impulse response functions and then convolved with the canonical haemodynamic response function (Friston et al., 1994). Linear contrasts were defined to test specific effects. Time derivatives were included in the basis functions set. Following smoothness estimation (Kiebel et al., 1999), linear contrasts of parameter estimates were defined to test the specific effects of each condition with each individual dataset. Voxel values for each contrast resulted in a statistical parametric map of the corresponding $t$-statistic, which was then transformed into the unit normal distribution (SPM Z). The statistical parametric maps from each individual dataset were then entered into second-level, random effects analyses accounting for both scan-to-scan and subject-to-subject variability. More precisely, the sets of individual statistical maps corresponding to a specific effect of interest were entered as covariates in multiple regression models (ANOVA without a constant) as implemented in SPM2 and the corresponding group effects were assessed by applying linear contrasts (again following smoothness estimation) to the (second-level) 
parameter estimates generating a $t$-statistics map for each group effect of interest. The correlation analyses of the fMRI blood oxygenation level-dependent (BOLD) signal with given parameters of interest (e.g. the pleasantness ratings) were performed at the second level by applying one-sample $t$-tests to the first-level $t$-maps resulting from performing linear parametric modulation as implemented in SPM2. Unless otherwise stated, reported $P$-values for each cluster based on this group analysis are fully corrected (fc) for the number of comparisons (resels) in the entire volume ('whole-brain' multiple comparisons, Worsley et al., 1996). Peaks are reported for which $P<0.05$. For brain regions where there was a prior hypothesis as described in the Introduction and Materials and methods, namely in the parts of the orbitofrontal cortex, pregenual cingulate cortex, ventral striatum and anterior insula in which we and others have found activations in previous studies to the taste, smell, sight and/or texture of food (O'Doherty et al., 2001, 2002; de Araujo et al., 2003a,b; de Araujo \& Rolls, 2004; Kringelbach \& Rolls, 2004; Rolls, 2005, 2006), we used small volume corrections (svcs). These activations correspond to voxels significant when corrected for the number of comparisons made within each region (svc applied with a sphere of $8 \mathrm{~mm}$ chosen to be greater than or equal to the spatial smoothing kernel) (Worsley et al., 1996). Peaks with $P<0.05$ false discovery rate (FDR)corrected across the small volume were considered significant. In addition, peaks are reported that survive a threshold of $P<0.005$ uncorrected if in the predicted regions outlined in our hypotheses. The percent change in the BOLD signal for different stimuli within regions of interest identified from the contrast analyses was extracted using the SPM Marsbar toolbox documented at http://marsbar.sourceforge.net/.

\section{Results}

\section{Ratings}

The ratings of pleasantness, intensity and wanting of the flavour obtained during the scanning in each trial in the cravers and noncravers are shown in Fig. 1. For all of the ratings, there were significant main effects $(P<0.001)$ of subject group (craver vs. noncraver), reflecting the lower ratings of the non-cravers, and significant main effects $(P<0.001)$ of stimulus type. In pre-planned comparisons, it was shown that the pleasantness ratings for chocolate in the mouth with the sight of chocolate were higher for cravers than noncravers (chocd, $t=2.29$, d.f. $=14, P=0.038$ ), but not for the condensed milk (cmilk, $t=1.46$, d.f. $=14, P=0.17$ ). The condensed milk thus acts as a non-chocolate, control, comparison condition and, as shown in Fig. 1, was liked by the cravers less than the chocolate. [Indeed, post-hoc tests following a significant $(P<0.004)$ one-way ANOVA within the cravers showed that the condensed milk was significantly less pleasant than every one of the chocolate stimuli, with $P$-values between 0.001 and 0.029.] The ratings of pleasantness and wanting, which may in part reflect different consummatory and appetitive processes (Berridge \& Robinson, 2003), obtained in every trial to a stimulus were correlated with each other in the non-cravers $(r=0.57$, d.f. $=40, P=0.001)$ but not in the cravers $(r=0.16$, d.f. $=35, P=0.35)$. The ratings of pleasantness and intensity were correlated with each other in the cravers $(r=0.59$, d.f. $=35, P=0.0002)$ and less so in the non-cravers $(r=0.31$, d.f. $=40, P=0.056)$.

For the functional imaging results, we first consider the main effects of chocolate in the mouth, to show the brain areas activated by this taste, texture and olfactory stimulus. We then show contrasts to analyse how the effects of chocolate are different in cravers vs. noncravers. The activations shown are minus the rinse control.

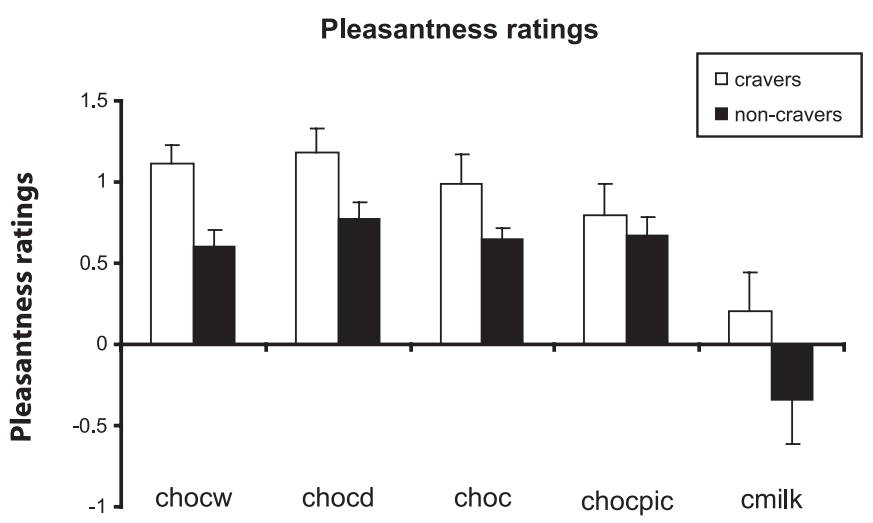

Intensity ratings

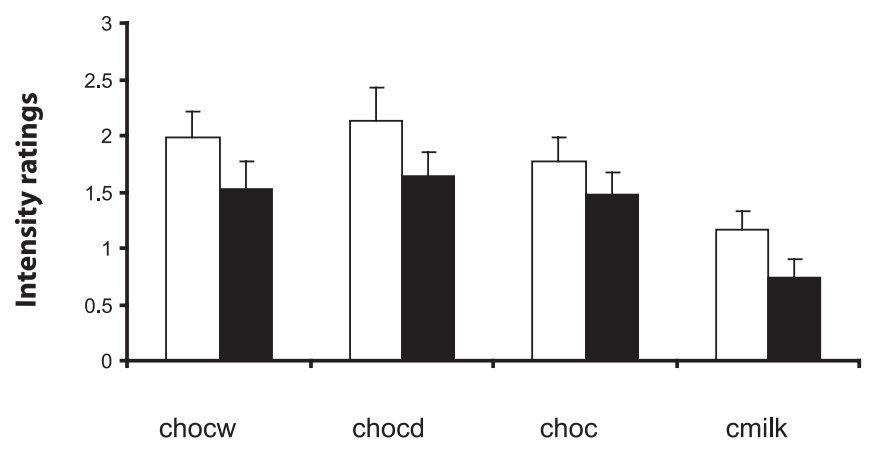

Wanting ratings

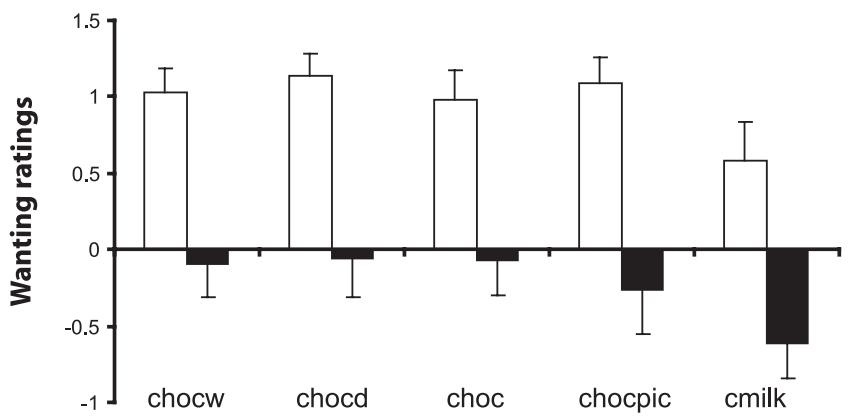

FIG. 1. The ratings of pleasantness, intensity and wanting (means \pm SEM). choc, chocolate in the mouth only + grey visual stimulus; chocpic, picture of dark chocolate only; chocd, chocolate in the mouth + a picture of dark chocolate; chocw, chocolate in the mouth + a picture of white chocolate; cmilk, condensed milk in the mouth + grey visual stimulus. (See further Table 1.)

\section{Chocolate in the mouth}

As expected, the choc condition resulted in activation in the primary taste cortex $\left(\left[\begin{array}{lll}-30 & 12 & 0\end{array}\right] Z=5.62 \mathrm{fc}, P<0.001\right.$ and [32 220$] Z=5.25 \mathrm{fc}, P<0.001$ ). This is illustrated in Fig. 2, which shows a coronal plane image taken through the taste insula. The same section shows some activation in the dorsal part of the anterior cingulate cortex ([ $\left.\begin{array}{lll}-4 & 24 & 38\end{array}\right] Z=4.48$ fc, $\left.P<0.001\right)$. Activations were also found in the mid-orbitofrontal cortex ([-20 42 -4] $Z=3.56$ svc, $P<0.003$ ) and in a region to which it projects, the ventral striatum $\left(\left[\begin{array}{lll}-12 & 6 & 4\end{array}\right] Z=4.47 \mathrm{fc}, P<0.001\right)$. There was also activation in the dorsolateral prefrontal cortex 


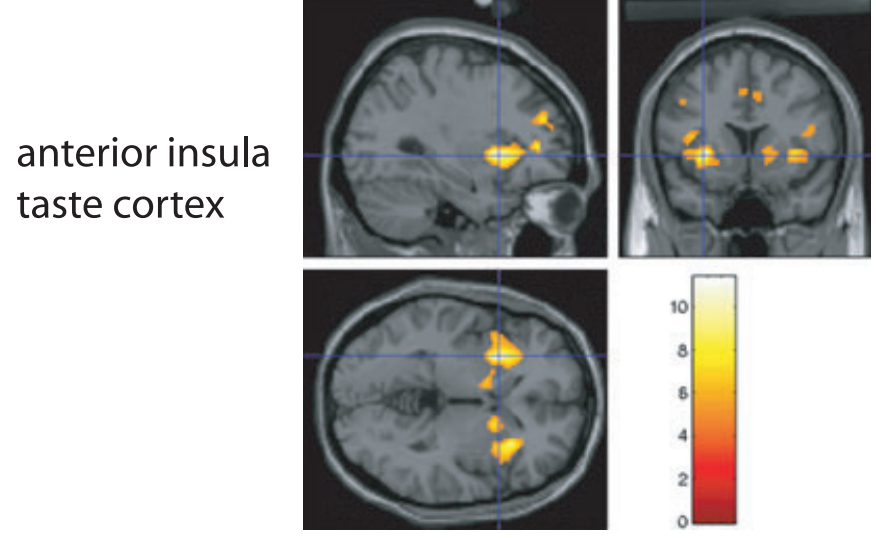

FIG. 2. The effects of chocolate in the mouth, across all subjects; activation is shown in the primary taste cortex in the anterior insula, e.g. at $\left[\begin{array}{lll}-30 & 12 & 0\end{array}\right]$ and $\left[\begin{array}{lll}32 & 22 & 0\end{array}\right]$ ( fc, $\left.P<0.001\right)$. The coronal section shows some activation in the dorsal part of the anterior cingulate cortex (centred at $\left[\begin{array}{lll}-4 & 24 & 38\end{array}\right] \mathrm{fc}$, $P<0.001)$

([-30 44 26] $Z=4.59$ fc, $P<0.001$ ), a region known to be activated by taste (Kringelbach et al., 2004).

\section{Chocolate in the mouth only: cravers vs. non-cravers}

The criterion level for a significant difference between cravers and noncravers in the brain activations to chocolate in the mouth in the condition in which no chocolate was shown was not reached, although there was extensive bilateral medial orbitofrontal cortex activation showing a larger BOLD signal in the cravers than the non-cravers ([ $\left.\begin{array}{lll}-6 & 32 & -30\end{array}\right]$ $Z=2.31, P=0.01$ uncorrected, i.e. below the criterion). The implication is that when significant differences between cravers and noncravers in the brain responses to chocolate were found, as described below, an important factor is the sight of the chocolate, which was present in the other conditions. Moreover, we show below that chocolate in the mouth can contribute to a greater than linear summation effect when combined with the sight of chocolate.

\section{Chocolate in the mouth with the sight of chocolate: cravers vs. non-cravers}

We now consider the effect of showing a picture of the dark chocolate while chocolate is in the mouth (chocd). More activation was found in the cravers than in the non-cravers in the anterior cingulate cortex ( $\left[\begin{array}{lll}-8 & 22 & 28\end{array}\right] Z=3.04 \mathrm{svc}, P<0.03$ ) and in the pregenual cingulate cortex ([4 30 8] $Z=2.85$ svc, $P<0.05$ ), as illustrated in Fig. 3 . Figure 3 also shows more activation in the ventral striatum in the cravers, but this did not reach significance.

\section{The sight of chocolate: cravers vs. non-cravers}

We now consider the effect of showing only a picture of the dark chocolate (chocpic). The sight of chocolate produced more activation of mid and medial parts of the orbitofrontal cortex in cravers than noncravers (e.g. [-28 42-10] $Z=2.92$ svc, $P<0.05$ ), as shown in Fig. $4 \mathrm{a}$. The time course for the sight of chocolate condition (chocpic, Fig. 4b) shows the greater activation in this orbitofrontal cortex region and also, interestingly, that the same region is also activated by chocolate in the mouth (choc, Fig. 4b). Thus, this orbitofrontal cortex region is multimodal. The sight of chocolate also activated the ventral

\section{Chocolate in the mouth with sight of dark chocolate :cravers vs non-cravers}
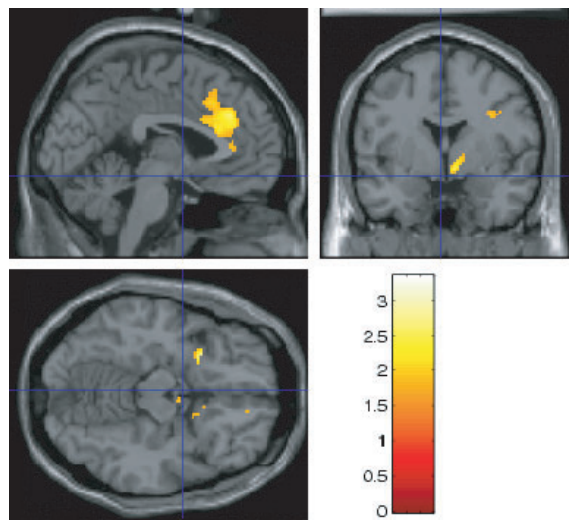

FIG. 3. Chocolate in the mouth and the sight of dark chocolate. More activation was found in the cravers than in the non-cravers in the anterior cingulate cortex ([ $\left[\begin{array}{lll}-8 & 22 & 28\end{array}\right]$ small volume correction (svc), $\left.P<0.03\right)$ and the pregenual cingulate cortex ([ $\left.\left.\begin{array}{lll}4 & 30 & 8\end{array}\right] \mathrm{svc}, P<0.05\right)$. Also shows activation in the ventral striatum.

striatum ([-4 $16-12]) Z=2.66, P=0.004$ uncorrected) more in cravers than non-cravers. The time course for the ventral striatum in Fig. $4 \mathrm{~d}$ shows the greater activation in cravers than non-cravers by the sight of chocolate.

\section{Supralinearity: the sight and flavour of chocolate compared with the sum of the activations to the sight alone and the flavour alone}

To investigate whether the combination of the sight and flavour of chocolate might be especially effective in cravers, we performed a supralinearity analysis, comparing activations to a combination of the sight and flavour of chocolate with the sum of the activations to the sight alone and the flavour alone, and tested whether this supralinearity was greater in the cravers than in the non-cravers. We found greater supralinearity in the pregenual cingulate cortex ([-2 304$]$ $Z=2.91 \mathrm{svc}, P=0.05)$ in the cravers relative to the non-cravers, as illustrated in Fig. 5. The time course of the effect to the combination (chocd) is illustrated in Fig. 4b. We also found greater supralinearity in

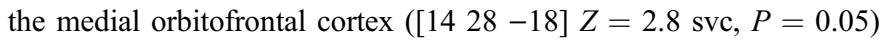
and in the head of the caudate nucleus ( $\left[\begin{array}{lll}-10 & 2 & 14\end{array}\right] Z=2.81 \mathrm{svc}$, $P=0.03)$. We note that the orbitofrontal cortex projects to both the pregenual cingulate cortex and the head of the caudate nucleus. These findings show that the flavour of chocolate in the mouth, when combined with the sight of chocolate, can have greater effects in cravers than in non-cravers.

\section{Effects of the sight of dark vs. white chocolate in cravers vs. non-cravers}

Chocolate cravers may be especially responsive to the sight of a rich, fine, dark chocolate as opposed to the sight of white chocolate. We accordingly tested whether a contrast of chocd-chocw was different in cravers and non-cravers. A significantly larger difference between the activations to dark vs. white chocolate was found in cravers relative to non-cravers in the anterior cingulate cortex ( $\left[\begin{array}{lll}-8 & 28 & 30\end{array}\right] Z=3.21 \mathrm{svc}$, $P=0.01)$ and some activation that did not reach significance was found in the pregenual cingulate cortex, as illustrated in Fig. 6. These 


\section{Sight of dark chocolate only: cravers vs non-cravers}

a

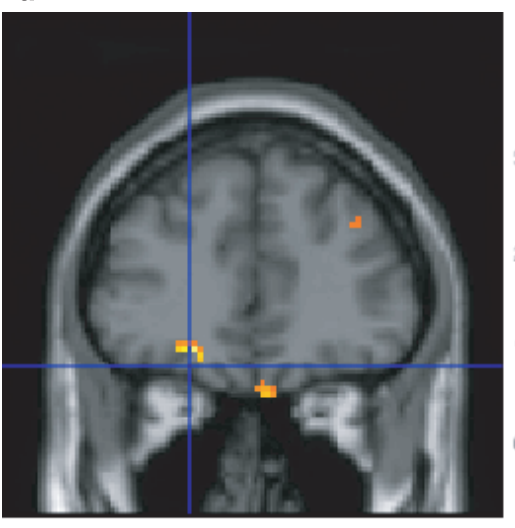

C

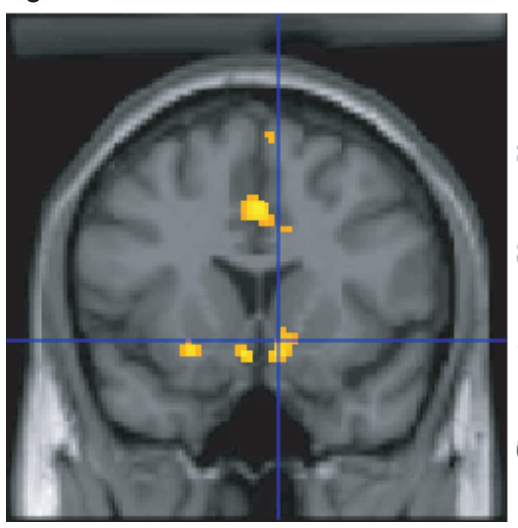

b

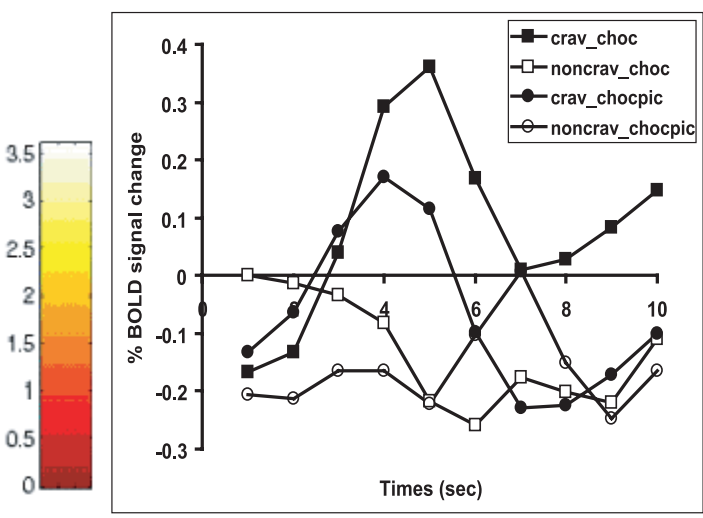

d

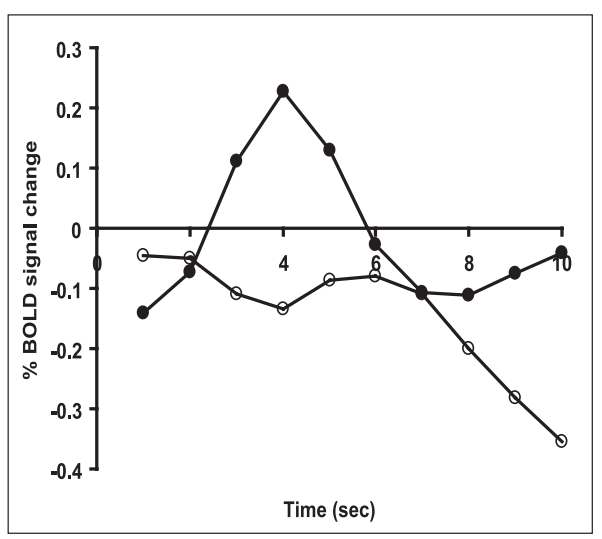

FIG. 4. (a) The sight of chocolate [picture of dark chocolate only (chocpic)] produced more activation of mid and medial parts of the orbitofrontal cortex in cravers than non-cravers (e.g. [ $-2842-10]$ small volume correction, $P<0.05$ ) with (b) the time courses for the chocpic and chocolate in the mouth only + grey visual stimulus (choc) conditions. (c) The sight of chocolate also produced more activation in the cravers than the non-cravers in the ventral striatum ([-4 16 -12] uncorrected, $P=0.004$ ) with (d) the time courses for the chocpic condition in the ventral striatum. BOLD, blood oxygenation level-dependent.

are areas that receive connections from the orbitofrontal cortex. Although there was no difference in the subjective ratings to these stimuli (see Fig. 1), we note that brain activations may occur, and might even influence behavioural choice, in the absence of subjective awareness (Rolls, 2005).

\section{Interaction effects: brain regions where chocolate vs. milk produced larger effects in cravers than non-cravers}

This interaction effect was tested in a statistical design in which the covariates were the effects of chocd in cravers, the effects of chocd in non-cravers, the effects of cmilk in cravers and the effects of cmilk in non-cravers. The interaction term testing for where the chocolate condition relative to the milk condition produced significantly greater effects in cravers than in non-cravers showed significant effects in the posterior orbitofrontal cortex/agranular insula transition area at [34 $14-8] \quad(Z=2.63, P=0.004$ uncorrected $)$ and this was seen bilaterally.

\section{Correlation with pleasantness ratings}

We now consider the correlations with the subjective ratings. The sites where correlations are found with, for example, pleasantness help in the interpretation of some of the effects described above in, for example, the medial orbitofrontal cortex and pregenual cingulate cortex. The ratings used for all of the correlation analyses were choc, chocd, chocw and cmilk (see Table 1), as oral stimulus delivery was involved in all of these cases.

In Fig. 7a we show where significantly higher correlations with the pleasantness ratings in the cravers vs. the non-cravers are found in the pregenual cingulate cortex ([-2 40 2 $]$ C $Z=3.34$ svc, $P=0.01)$ extending down into the medial orbitofrontal cortex ([8 $36-8] \quad Z=2.52$ svc, $P<0.05$ ) and in the dorsolateral prefrontal cortex at [30 42 28] $(Z=3.83 \mathrm{fc}, P<0.001)$, as also shown in Fig. 7a. Consistent with this, in the group of cravers there was a significant correlation with the pleasantness ratings in the medial orbitofrontal cortex $\left(\left[\begin{array}{lll}-8 & 28 & -20]\end{array}=3.88\right.\right.$ fc, $\left.P=0.008\right)$ and in the pregenual cingulate cortex ([- $\left.\begin{array}{llll}-6 & 56 & 16\end{array}\right] Z=2.7 \mathrm{svc}$, $P=0.01)$, with smaller effects in the group of non-cravers in the medial orbitofrontal cortex $\left(\left[\begin{array}{lll}-2 & 24 & -28\end{array}\right] Z=2.81\right.$ svc, $\left.P=0.03\right)$. The relation between the percentage change in the BOLD stimuli and the subjective ratings is shown in Fig. $7 \mathrm{~b}$. It is noticeable that there is a greater range in the BOLD signal in the cravers than the non-cravers in the pregenual cingulate cortex and the dorsolateral prefrontal cortex. In the medial orbitofrontal cortex of cravers there is little variance in the BOLD signal for any given rating, i.e. the rating is very closely related to the BOLD signal. 


\section{Supralinearity: Chocd $>$ (choc \& chocpic) cravers vs non-cravers}
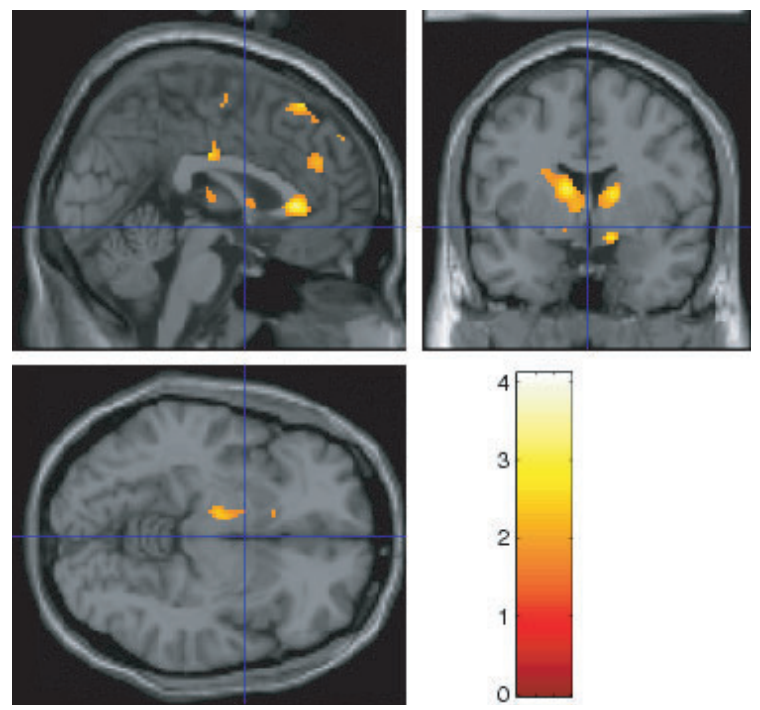

FIG. 5. Supralinearity analysis showing where there was more supalinearity (where the activations to a combination of the sight and flavour of chocolate were larger than the sum of the activations to the sight alone and the flavour alone) in the cravers than in the non-cravers. Greater supralinearity is shown in the pregenual cingulate cortex ([-2 304$]$ small volume correction (svc), $P=0.05)$ and also in the head of caudate $\left(\left[\begin{array}{lll}-10 & 2 & 14\end{array}\right]\right.$ svc, $\left.P=0.03\right)$.

\section{Sight of dark chocolate compared to white chocolate : cravers vs non-cravers}
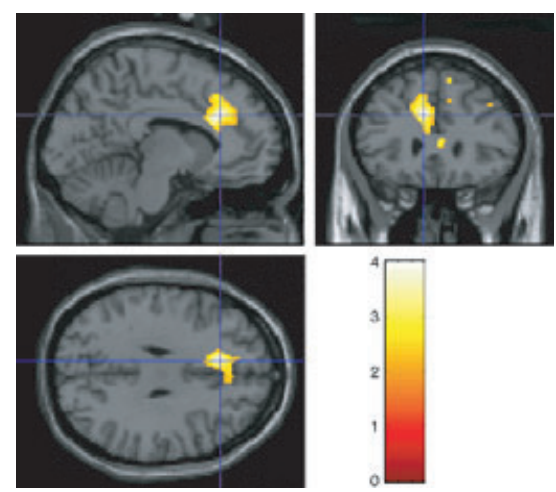

FIG. 6. Contrast of the sight of dark chocolate vs. sight of white chocolate in cravers compared with non-cravers. Significant activations were found in the dorsal part of the anterior cingulate cortex at $\left[\begin{array}{lll}-8 & 28 & 30\end{array}\right]$ (small volume correction, $P=0.01)$. Coronal section also shows some non-significant activation in the pregenual cingulate cortex.

FIG. 7. (a) Correlations with the pleasantness ratings in the cravers vs. the non-cravers are shown in the pregenual cingulate cortex ([-2 40 2 2$]$ small volume correction (svc), $P=0.01)$ extending down into the medial orbitofrontal cortex at $[836-8]$ (svc, $P<0.05)$ and in the dorsolateral prefrontal cortex ([30 4228$]$ fc, $P<0.001$ ). (b) Relation between percent blood oxygenation level-dependent (BOLD) change and pleasantness ratings for the pregenual cingulate cortex, medial orbitofrontal cortex and dorsolateral prefrontal cortex in cravers and non-cravers. Means \pm SEM are shown.

\section{Correlation with intensity ratings}

The intensity ratings were highly correlated in the cravers with activations in the pregenual cingulate cortex $\left(\left[\begin{array}{lll}0 & 40 & -2\end{array}\right] Z=3.13 \mathrm{svc}\right.$, $P=0.02)$ and the medial orbitofrontal cortex $\left(\left[\begin{array}{lll}6 & 38 & -10]\end{array}\right]=3.08\right.$

a

Correlation with the pleasantness of the chocolate related stimuli : cravers vs non-cravers
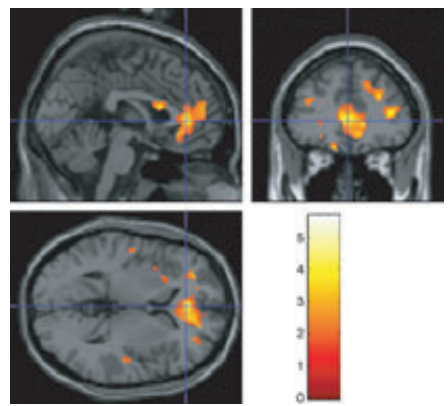

b \% BOLD change as a function of pleasantness ratings in the Pregenual cingulate cortex

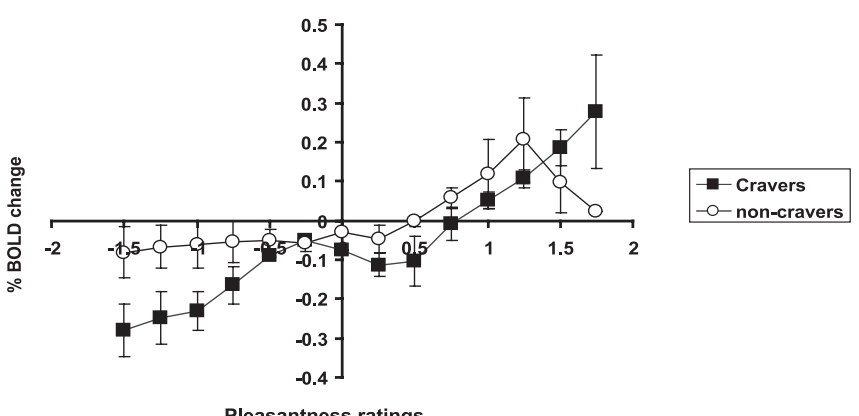

Pleasantness ratings

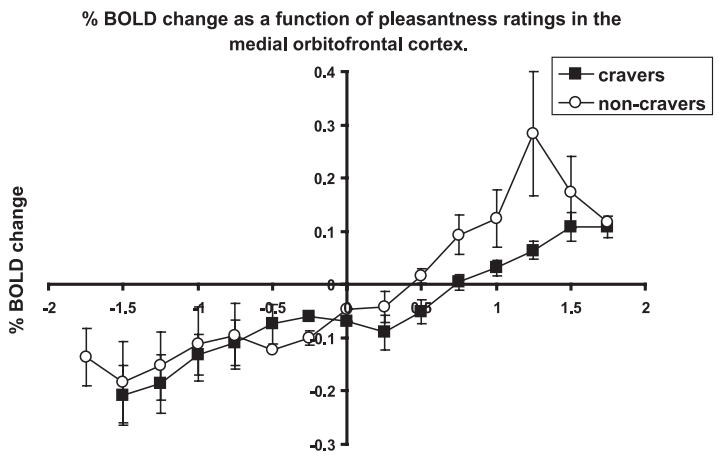

Pleasantness ratings

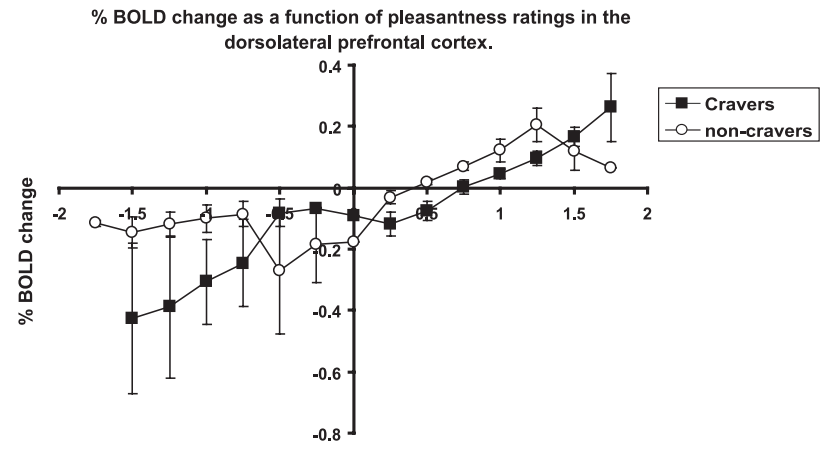

Pleasantness ratings 


\section{Correlation with the intensity of the chocolate related stimuli in the cravers}

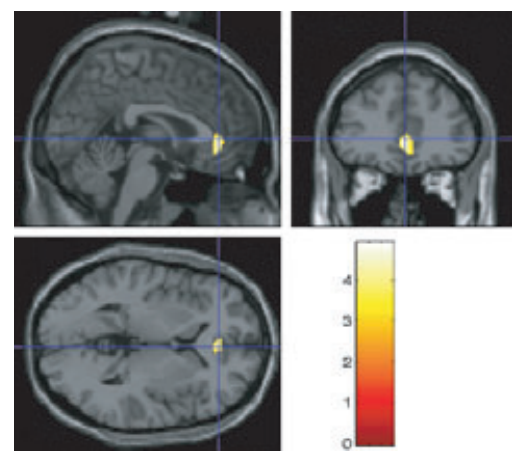

FIG. 8. Correlation with the intensity ratings in the cravers. Significant correlations were found in the pregenual cingulate cortex ([ $\left[\begin{array}{lll}0 & 40 & -2\end{array}\right]$ small volume correction, $P<0.02$ ) (illustrated) and in the medial orbitofrontal cortex ([6 38 -10] uncorrected, $P=0.001$ ).

uncorrected, $P=0.001$ ), as illustrated in Fig. 8. There were no significant correlations in these regions in the non-cravers and consistently a direct statistical comparison showed that the correlations were significantly higher in the cravers than the non-cravers in these regions (pregenual cingulate cortex) ([ [ $\left.\begin{array}{lll}4 & 38 & 2\end{array}\right] \quad Z=2.96$ svc, $P=0.03)$. To help interpret these correlations with the intensity ratings, we note that in this investigation most of the stimuli were chocolate or sweet (condensed milk) and the intensity ratings for this particular set of stimuli were, in fact, correlated with the pleasantness ratings $(r=0.52, P<0.001$ ) (a finding related to the absence of intense but very unpleasant stimuli in this stimulus set). Thus, the correlations with the intensity ratings may in this study reflect at least partly the subjective pleasantness of the stimuli.

\section{Correlation with ratings for the wanting for chocolate}

There was some correlation with the wanting ratings across all subjects in the medial orbitofrontal cortex ([10 26-10]) but this did not quite reach the criterion for significance $(Z=2.4, P=0.008$ uncorrected). No significant differences between the correlations with wanting of brain activations in the cravers vs. the non-cravers were found.

\section{Discussion}

No differences between chocolate cravers and non-cravers were found in the activations in the primary taste cortex in the anterior insula (see Fig. 2), although both taste and oral texture are represented in this region (de Araujo \& Rolls, 2004; Verhagen et al., 2004). Further, the activations in the primary taste cortex were not correlated with pleasantness, wanting or intensity ratings for chocolate. Thus, it was not differences in the primary taste cortex, or physical sensitivity to taste and oral texture, which separated the cravers from the noncravers.

Differences between cravers and non-cravers were found further on in processing, in brain structures such as the medial orbitofrontal cortex and two areas to which it projects (Ongur \& Price, 2000; Rolls, 2005), the pregenual cingulate cortex and ventral striatum. The medial orbitofrontal cortex was more activated in cravers than non-cravers by the sight of chocolate (Fig. 4a and b, chocpic) and by the combination of the sight of chocolate and chocolate in the mouth (chocd) than by the sum of the components (choc and chocpic) (Fig. 5). Thus, a key region that responds differently between cravers and non-cravers is the orbitofrontal cortex and its contribution is multimodal. Moreover, in that the activations were correlated with the pleasantness ratings for the set of stimuli (Fig. 7), the orbitofrontal cortex contribution can be related to the greater affective, hedonic responses of chocolate in cravers than non-cravers.

The pregenual/anterior cingulate cortex showed a greater activation in cravers than non-cravers to the combination of the sight and taste of chocolate (Fig. 3, chocd), and indeed the combination produced greater supralinear effects in cravers than in non-cravers (Fig. 5). Interestingly, there were no differences between cravers and noncravers, and indeed no strong activations in the pregenual cingulate, to just the sight of chocolate (chocpic) or just chocolate in the mouth (choc). The pregenual activations were correlated with the pleasantness (Fig. 7) and intensity (Fig. 8) but not wanting ratings. The implication is that the pregenual cingulate cortex may contribute especially just when all of the sensory aspects of the stimuli (sight and mouth feel) have combined to make a very effective stimulus that can then drive behaviour more in cravers than non-cravers.

The ventral striatum showed greater responses to the sight of chocolate in cravers than non-cravers (Fig. 4c and d). Interestingly, although across all subjects chocolate in the mouth produced strong activation in the ventral striatum, there were no differences between cravers and non-cravers. The implication is that the ventral striatum in cravers vs. non-cravers contributes especially to the conditioned, i.e. visual, component of chocolate craving. Interestingly, the head of the caudate nucleus, which like the ventral striatum receives from the orbitofrontal cortex, had a greater supralinearity for the combination of the sight and flavour of chocolate compared with the sum of the components in cravers vs. non-cravers (Fig. 5), indicating that conditioned (sight) and unconditioned (flavour) effects interact in the striatum.

An aim of this study was to investigate differences between individuals in their brain responses to foods to advance our understanding of the brain systems that contribute to the pleasantness (or liking) and wanting (or craving) for a food. We found that brain areas such as the orbitofrontal cortex and two areas to which it connects, the pregenual cingulate cortex and ventral striatum, are activated more by chocolate in cravers than in non-cravers. Given that food cravers (and chocolate cravers) are high in measures of sensitivity to reward (Davis et al., 2004; Franken \& Muris, 2005) and arousal produced by images of chocolate (Tuomisto et al., 1999; Rodriguez et al., 2005), the present study provides neurobiological evidence that it is these brain systems (and not for example the primary, insular, taste cortex) that react strongly to the rewarding properties of a craved food, such as chocolate, in cravers. A study by Beaver et al. (2006) showed that reward sensitivity in different individuals (as measured by a behavioural activation scale) is correlated with activations to pictures of appetizing vs. disgusting food but they made no attempt to measure brain activations produced by a food that is specifically craved, such as chocolate in a group of chocolate cravers, nor did they measure activations to the flavour of any foods. The present study shows in a direct comparison of chocolate cravers and non-cravers that is therefore directly related to craving, that differences in activations to the flavour of chocolate and/or the sight of chocolate are not found in areas involved in taste and oral texture such as the insular (primary) taste cortex but are found in areas including the orbitofrontal cortex, ventral striatum and pregenual cingulate cortex. Although Small et al. (2001) investigated brain activations to chocolate in a 
positron emission tomography study, they performed no comparison of chocolate cravers vs. non-cravers and thus their study does not address differences between cravers and non-cravers when shown or given a craved food. Their study also used water as a control and had no sight of chocolate condition. Further studies investigated brain responses to food but not between different groups of individuals. For example, in another positron emission tomography study, activation of the orbitofrontal cortex was found to food that was being tasted, smelled and tasted, and the metabolic response of the brain was correlated with the desire for the food (Wang et al., 2004). In another positron emission tomography study, it was shown that the orbitofrontal cortex was activated by high incentive restaurant menus (Arana et al., 2003). In an fMRI study, foodrelated visual stimuli elicited greater responses in the amygdala, parahippocampal gyrus and anterior fusiform gyrus when participants were in a hungry state relative to a satiated state (LaBar et al., 2001).

In the present study the greater activation of the ventral striatum by the sight of chocolate in cravers than non-cravers (Fig. 5) is of particular interest because the ventral striatum is strongly implicated in animals in the effects of conditioned incentives (of which one example is the sight of food and another is monetary reward) (Robbins \& Everitt, 1996; Rolls et al., 2007) and has been shown to be activated by conditioned environmental cues associated with chocolate (Schroeder et al., 2001). Similarly, the medial orbitofrontal cortex, which projects to the ventral striatum, was also more strongly activated in cravers than non-cravers by the sight of chocolate. It is of interest that the ventral striatum is implicated in craving for other reinforcers and in the reinstatement of craving produced by conditioned, e.g. visual, stimuli (Robbins \& Everitt, 1996; Kelley, 2003). Further, the orbitofrontal cortex is also a site implicated in the rewarding effects of other reinforcers (Phillips et al., 1981; Völlm et al., 2004) and is involved in the rapid association learning between visual stimuli and reinforcers (Rolls et al., 1996; Hornak et al., 2004; Rolls, 2005). The present study thus provides new evidence that these brain regions are also involved in the effects of visual stimuli on food craving and emphasize the importance of these brain regions in making food stimuli attractive. The findings underlie the importance of conditioned cues, such as the sight of chocolate, in producing different effects in different individuals, in brain regions that we now understand to include the orbitofrontal cortex and ventral striatum. Such cues are likely to be important in food choice, by making a craved food particularly pleasant. The findings indicate that these brain systems that respond to the conditioned incentive value of food have larger responses in cravers than in non-cravers to food and the implication is that part of the difference between cravers and non-cravers is that the chocolate cravers are more responsive to the conditioned incentive value of the craved food. Although the body mass index of the cravers and the non-cravers was not different in this study, and more generally food cravers are not necessarily overweight (Franken \& Muris, 2005) (perhaps because of cognitive control), this study did provide new evidence that individual differences in brain activations to a craved food may be linked to the consumption of that food, in that in this study the chocolate cravers ate more chocolate than non-cravers (370 vs. $22 \mathrm{~g} /$ week) and ate chocolate more frequently (see Materials and methods).

The findings described here offer a new view on the brain mechanisms that underlie the increased sensitivity to craved foods in cravers. The results suggest that sensory systems such as the primary taste cortex and inferior temporal visual cortex are not different in their responsiveness to chocolate in cravers and non-cravers, and that the first cortical area that is hyper-reactive to craved foods in cravers is the orbitofrontal cortex (which receives direct inputs from the insular taste cortex and inferior temporal visual cortex). The orbitofrontal cortex could then, with its onward connections to the ventral striatum and pregenual cingulate cortex, produce the greater activations found in the ventral striatum and pregenual cingulate to craved foods. This would imply that at least part of the driver towards craving is heightened responsiveness in the orbitofrontal cortex in cravers and that any altered activity in the dopamine system may be secondary to this, and indeed could be produced by the downward projections from the striatum to the dopamine neurones in the midbrain (Rolls, 2005, 2008).

In conclusion, this study shows how brain processing for craved foods is different between people, and is related to their liking and craving for that food and the amount of that food eaten. These brain differences are likely to be important for understanding not only how different people respond to highly palatable foods but also how these differences are related to food choice, food craving and the amount of specific foods eaten by different individuals.

\section{Abbreviations}

BOLD, blood oxygenation level-dependent; choc, chocolate in the mouth only plus grey visual stimulus; chocd, chocolate in the mouth plus a picture of dark chocolate; chocpic, picture of dark chocolate only; chocw, chocolate in the mouth plus a picture of white chocolate; cmilk, condensed milk in the mouth plus grey visual stimulus; fc, fully corrected; fMRI, functional MRI; SPM, statistical parametric mapping; svc, small volume correction.

\section{References}

Arana, F.S., Parkinson, J.A., Hinton, E., Holland, A.J., Owen, A.M. \& Roberts, A.C. (2003) Dissociable contributions of the human amygdala and orbitofrontal cortex to incentive motivation and goal selection. J. Neurosci., 23, 9632-9638.

de Araujo, I.E.T. \& Rolls, E.T. (2004) The representation in the human brain of food texture and oral fat. J. Neurosci., 24, 3086-3093.

de Araujo, I.E.T., Kringelbach, M.L., Rolls, E.T. \& Hobden, P. (2003a) The representation of umami taste in the human brain. J. Neurophysiol., 90, $313-$ 319.

de Araujo, I.E.T., Kringelbach, M.L., Rolls, E.T. \& McGlone, F. (2003b) Human cortical responses to water in the mouth, and the effects of thirst. J. Neurophysiol., 90, 1865-1876.

Beaver, J.D., Lawrence, A.D., Ditzhuijzen, J.V., Davis, M.H., Woods, A. \& Calder, A.J. (2006) Individual differences in reward drive predict neural responses to images of food. J. Neurosci., 26, 5160-5166.

Berridge, K.C. \& Robinson, T.E. (2003) Parsing reward. Trends Neurosci., 26, $507-513$

Cardinal, N., Parkinson, J.A., Hall, J. \& Everitt, B.J. (2002) Emotion and motivation: the role of the amygdala, ventral striatum, and prefrontal cortex. Neurosci. Biobehav. Rev., 26, 321-352.

Cepeda-Benito, A., Fernandez, M.C. \& Moreno, S. (2003) Relationship of gender and eating disorder symptoms to reported cravings for food: construct validation of state and trait craving questionnaires in Spanish. Appetite, 40, 47-54.

Collins, D.L., Neelin, P., Peters, T.M. \& Evans, A.C. (1994) Automatic 3D intersubject registration of MR volumetric data in standardized Talairach space. J. Comput. Assist. Tomogr., 18, 192-205.

Critchley, H.D. \& Rolls, E.T. (1996) Hunger and satiety modify the responses of olfactory and visual neurons in the primate orbitofrontal cortex. J. Neurophysiol., 75, 1673-1686.

Davis, C., Strachan, S. \& Berkson, M. (2004) Sensitivity to reward: implications for eating and overweight. Appetite, 42, 131-138.

Everitt, B. (1997) Craving cocaine cues: cognitive neuroscience meets drug addiction research. Trends Cogn. Sci., 1, 1-2.

Franken, I.H.A. \& Muris, P. (2005) Individual differences in reward sensitivity are related to food craving and relative body weight in healthy women. Appetite, 45, 198-201. 
Friston, K.J., Worsley, K.J., Frackowiak, R.S.J., Mazziotta, J.C. \& Evans, A.C. (1994) Assessing the significance of focal activations using their spatial extent. Hum. Brain Mapp., 1, 214-220.

Friston, K.J., Glaser, D.E., Henson, R.N., Kiebel, S., Phillips, C. \& Ashburner, J. (2002) Classical and Bayesian inference in neuroimaging: applications. Neuroimage, 16, 484-512.

Gottfried, J.A., Small, D.M. \& Zald, D.H. (2006) The chemical senses. In Zald, D.H. \& Rauch, S.L. (Eds), The Orbitofrontal Cortex. Oxford University Press, Oxford, pp. 125-171.

Hornak, J., O’Doherty, J., Bramham, J., Rolls, E.T., Morris, R.G., Bullock, P.R. $\&$ Polkey, C.E. (2004) Reward-related reversal learning after surgical excisions in orbitofrontal and dorsolateral prefrontal cortex in humans. J. Cogn. Neurosci., 16, 463-478.

Kelley, A.E. (2003) Ventral striatal control of appetitive motivation: role in ingestive behavior and reward-related learning. Neurosci. Biobehav. Rev., 27, 765-776.

Kiebel, S.J., Poline, J.B., Friston, K.J., Holmes, A.P. \& Worsley, K.J. (1999) Robust smoothness estimation in statistical parametric maps using standardized residuals from the general linear model. Neuroimage, 10, 756-766.

Kringelbach, M.L. \& Rolls, E.T. (2004) The functional neuroanatomy of the human orbitofrontal cortex: evidence from neuroimaging and neuropsychology. Prog. Neurobiol., 72, 341-372.

Kringelbach, M.L., O’Doherty, J., Rolls, E.T. \& Andrews, C. (2003) Activation of the human orbitofrontal cortex to a liquid food stimulus is correlated with its subjective pleasantness. Cereb. Cortex, 13, 1064-1071.

Kringelbach, M.L., De Araujo, I.E.T. \& Rolls, E.T. (2004) Taste-related activity in the human dorsolateral prefrontal cortex. Neuroimage, 21, 781-788.

LaBar, K.S., Gitelman, D.R., Parrish, T.B., Kim, Y.H., Nobre, A.C. \& Mesulam, M.M. (2001) Hunger selectively modulates corticolimbic activation to food stimuli in humans. Behav. Neurosci., 115, 493-500.

McCabe, C. \& Rolls, E.T. (2007) Umami: a delicious flavor formed by convergence of taste and olfactory pathways in the human brain. Eur. J. Neurosci., 25, 1855-1864.

Michener, W. \& Rozin, P. (1994) Pharmacological versus sensory factors in the satiation of chocolate craving. Physiol. Behav., 56, 419-422.

O’Doherty, J., Rolls, E.T., Francis, S., Bowtell, R., McGlone, F., Kobal, G., Renner, B. \& Ahne, G. (2000) Sensory-specific satiety related olfactory activation of the human orbitofrontal cortex. Neuroreport, 11, 893-897.

O’Doherty, J., Rolls, E.T., Francis, S., Bowtell, R. \& McGlone, F. (2001) The representation of pleasant and aversive taste in the human brain. J. Neurophysiol., 85, 1315-1321.

O’Doherty, J.P., Deichmann, R., Critchley, H.D. \& Dolan, R.J. (2002) Neural responses during anticipation of a primary taste reward. Neuron, 33, 815826.

Ongur, D. \& Price, J.L. (2000) The organisation of networks within the orbital and medial prefrontal cortex of rats, monkeys and humans. Cereb. Cortex, 10, 206-219.

Phillips, A.G., Mora, F. \& Rolls, E.T. (1981) Intra-cerebral self-administration of amphetamine by rhesus monkeys. Neurosci. Lett., 24, 81-86.

Pritchard, T.C., Macaluso, D.A. \& Eslinger, P.J. (1999) Taste perception in patients with insular cortex lesions. Behav. Neurosci., 113, 663-671.

Robbins, T.W. \& Everitt, B. (1996) Neurobehavioural mechanisms of reward and motivation. Curr. Opin. Neurobiol., 6, 228-236.

Rodriguez, S., Fernandez, M.C., Cepeda-Benito, A. \& Vila, J. (2005) Subjective and physiological reactivity to chocolate images in high and low chocolate cravers. Biol. Psychol., 70, 9-18.
Rodriguez, S., Warren, C.S., Moreno, S., Cepeda-Benito, A., Gleaves, D.H., Del Carmen Fernandez, M. \& Vila, J. (2007) Adaptation of the food-craving questionnaire trait for the assessment of chocolate cravings: Validation across British and Spanish women. Appetite, 49, 245-250.

Rolls, E.T. (2005) Emotion Explained. Oxford University Press, Oxford.

Rolls, E.T. (2006) Brain mechanisms underlying flavour and appetite. Phil. Trans. R. Soc. Lond. B, 361, 1123-1136.

Rolls, E.T. (2007a) Sensory processing in the brain related to the control of food intake. Proc. Nutr. Soc., 66, 96-112.

Rolls, E.T. (2007b) Understanding the mechanisms of food intake and obesity. Obesity Rev., 8, 67-72.

Rolls, E.T. (2008) Memory, Attention, and Decision-Making: A Unifying Computational Neuroscience Approach. Oxford University Press, Oxford.

Rolls, E.T., Sienkiewicz, Z.J. \& Yaxley, S. (1989) Hunger modulates the responses to gustatory stimuli of single neurons in the caudolateral orbitofrontal cortex of the macaque monkey. Eur. J. Neurosci., 1, 53-60.

Rolls, E.T., Critchley, H.D., Mason, R. \& Wakeman, E.A. (1996) Orbitofrontal cortex neurons: role in olfactory and visual association learning. J. Neurophysiol., 75, 1970-1981.

Rolls, E.T., Critchley, H.D., Browning, A.S., Hernadi, A. \& Lenard, L. (1999) Responses to the sensory properties of fat of neurons in the primate orbitofrontal cortex. J. Neurosci., 19, 1532-1540.

Rolls, E.T., McCabe, C. \& Redoute, J. (2007) Expected value, reward outcome, and temporal difference error representations in a probabilistic decision task. Cereb. Cortex, in press. [doi:10.1093/cercor/bhm1097]

Schroeder, B.E., Binzak, J.M. \& Kelley, A.E. (2001) A common profile of cortical activation following exposure to nicotine- or chocolate-associated contextual cues. Neuroscience, 105, 535-545.

Small, D.M., Zatorre, R.J., Dagher, A., Evans, A.C. \& Jones-Gotman, M. (2001) Changes in brain activity related to eating chocolate: from pleasure to aversion. Brain, 124, 1720-1733.

Tuomisto, T., Hetherington, M.M., Morris, M.F., Tuomisto, M.T., Turjanmaa, V. \& Lappalainen, R. (1999) Psychological and physiological characteristics of sweet food 'addiction'. Int. J. Eating Disord., 25, 169-175.

Verhagen, J.V., Kadohisa, M. \& Rolls, E.T. (2004) The primate insular/opercular taste cortex: neuronal representations of the viscosity, fat texture, grittiness, temperature and taste of foods. J. Neurophysiol., 92 , $1685-1699$

Völlm, B.A., de Araujo, I.E.T., Cowen, P.J., Rolls, E.T., Kringelbach, M.L., Smith, K.A., Jezzard, P., Heal, R.J. \& Matthews, P.M. (2004) Methamphetamine activates reward circuitry in drug naïve human subjects. Neuropsychopharmacology, 29, 1715-1722.

Wang, G.J., Volkow, N.D., Telang, F., Jayne, M., Ma, J., Rao, M., Zhu, W., Wong, C.T., Pappas, N.R., Geliebter, A. \& Fowler, J.S. (2004) Exposure to appetitive food stimuli markedly activates the human brain. Neuroimage, 21, 1790-1797.

Wilson, J.L., Jenkinson, M., Araujo, I.E.T., Kringelbach, M.L., Rolls, E.T. \& Jezzard, P. (2002) Fast, fully automated global and local magnetic field optimisation for fMRI of the human brain. Neuroimage, 17, 967976.

Worsley, K.J., Marrett, P., Neelin, A.C., Friston, K.J. \& Evans, A.C. (1996) A unified statistical approach for determining significant signals in images of cerebral activation. Hum. Brain Mapp., 4, 58-73.

Yaxley, S., Rolls, E.T. \& Sienkiewicz, Z.J. (1988) The responsiveness of neurons in the insular gustatory cortex of the macaque monkey is independent of hunger. Physiol. Behav., 42, 223-229. 